Dear author,

Please note that changes made in the online proofing system will be added to the article before publication but are not reflected in this PDF.

We also ask that this file not be used for submitting corrections. 


\title{
Echocardiography-Guided False Lumen Catheterization for "Cheese-Wire" Technique in Complicated Type A Aortic Dissection
}

\author{
Hozan Mufty, Steffen Rex, Inge Fourneau, ${ }^{1}$ and Geert Maleux, ${ }^{3}$ Leuven, Belgium
}

Endovascular treatment of both type A and type B aortic dissections complicated by organ malperfusion is gaining increasing interest and evidence. Different fenestration techniques of the dissection membrane have already been described.

We present a 53-year-old patient with complicated type A aortic dissection after previous repair of the ascending aorta. The patient was treated with dissection membrane fenestration and "cheese-wire" technique. Owing to the mobility of the dissection flap, we encountered problems to perforate the dissection membrane with the use of a needle. Finally, on transesophageal echocardiography (TEE), a small dynamic dissection flap was visualized in the aortic arch, which could be catheterized by TEE for guidance. Downward traction of the through-wire caused infrarenal intima accumulation. lliac outflow obstruction could be solved by placement of a bare-metal stent.

Extensive screening for dynamic dissection of (re)-entry holes with the use of TEE is a valuable auxiliary in case needle perforation is difficult to achieve in complicated type A or B dissections.

\section{INTRODUCTION}

End-organ malperfusion is one of the most feared complications after acute aortic dissection. ${ }^{1}$ The approach of treatment of this entity depends on the type of dissection. Recent guidelines advise thoracic endografting as a first-line treatment option in case of type B dissection (class I; level of evidence, C). Endovascular fenestration should be

\footnotetext{
${ }^{1}$ Department of Vascular Surgery, University Hospitals Leuven, Leuven, Belgium.

${ }^{2}$ Department of Anesthesiology, University Hospitals Leuven and Department of Cardiovascular Science, KU Leuven, Leuven, Belgium.

${ }^{3}$ Department of Radiology, University Hospitals Leuven, Leuven Belgium.

Correspondence to: Hozan Mufty, MD, FEBVS, Department of Vascular Surgery, University Hospitals Leuven, Herestraat 49, 3000 Leuven, Belgium; E-mail:hozan.mufty@uzleuven.be

Ann Vasc Surg 2019; $\mathbf{\square}: 1-4$

https://doi.org/10.1016/i.avsg.2018.08.085

(c) 2018 Elsevier Inc. All rights reserved.

Manuscript received: June 29, 2018; manuscript accepted: August 7, 2018; published online:
}

considered in cases of persistent organ malperfusion after initial proximal repair (class IIa; level of evidence, C). ${ }^{2}$ In contrast, based on the current evidence, all patients with type A aortic dissection should be treated with surgery first. The operative outcome is highly influenced by the presence of persistent mesenteric malperfusion. In most cases, this malperfusion is caused by a combination of dynamic and static obstructions. Fenestration of the intimal flap is used in patients with dynamic malperfusion syndrome. ${ }^{3}$ In case standard catheterization of the false lumen fails, various techniques are described to perforate dissection membranes. $^{4-7}$ However, the dynamic state of the dissection membrane can create difficulties to perforate it. In this case, we encountered problems to perforate the dissection membrane with the use of needles (using transjugular intrahepatic portosystemic shunt (TIPS) and reentry catheter). On transesophageal echocardiography (TEE), we visualized a small dynamic dissection flap in the aortic arch, which could be catheterized by TEE for guidance. 
111

112

113

114

115

116

117

118

119

120

121

122

123

124

125

126

127

128

129

130

131

132

133

134

135

136

137

138

139

140

141

142

143

144

145

146

147

148

149

150

151

152

153
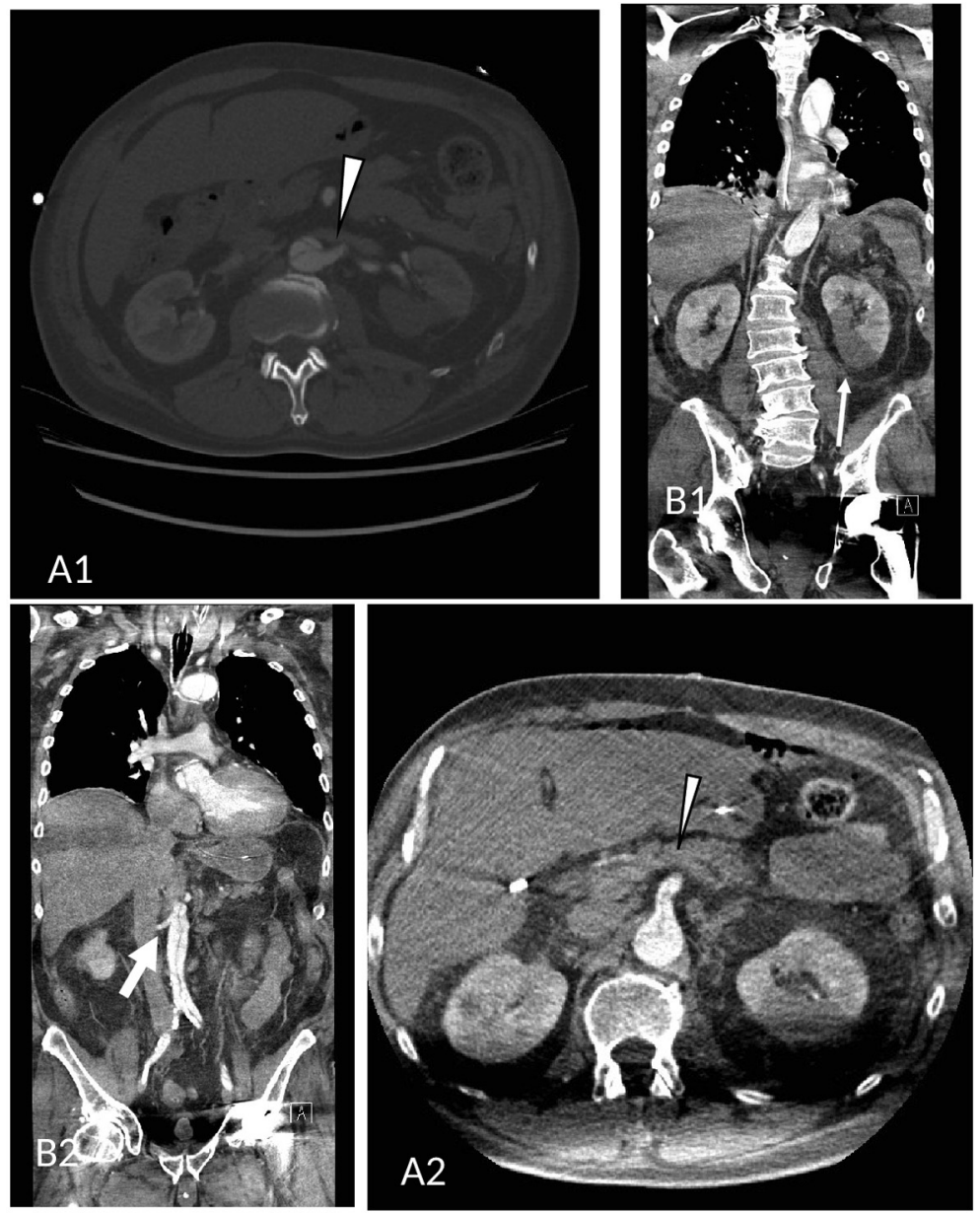

\section{A}

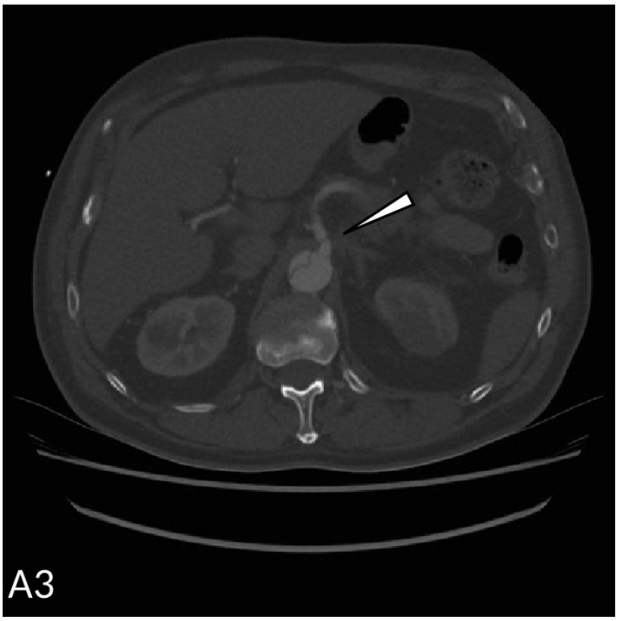

Fig. 1. Preoperative axial (A1 and A2) and reconstructed coronal (B1 and B2) computed tomography angiography images depicting the left renal artery originating from the false lumen (arrow head) (A1) with accompanying infarction of the left kidney lower pole

(small arrow) (B1), the right renal artery originating from the true lumen (large arrow) (B2), the superior mesenteric artery originating from both the true and false lumen (arrow head) (A2), and the celiac trunk originating from the false lumen (arrow head) (A3). because of complicated type A dissection with persisting visceral ischemia. Six weeks earlier, an open repair with Dacron interposition graft of the ascending aorta had been performed. The patient presented with recurrent thoracic pain and abdominal complaints. Computed tomography angiography (CTA) revealed progression of dissection into the visceral arteries with the celiac trunk and left renal artery originating from the false lumen, the right renal artery originating from the true lumen, and the superior mesenteric artery (SMA) originating from both lumina (Fig. 1). The SMA was occluded in the distal third. The left iliac artery originated from the false

Q3 lumen. Lactate, at the time of arrival in our hospital, was $4.1 \mathrm{mmol} / \mathrm{L}$, eGFR (Chronic Kidney Disease
Epidemiology Collaboration [CKD-EPI]) was $58 \mathrm{~mL} /$ $\min / 1.73 \mathrm{~m}^{2}$. Urgent explorative laparotomy was performed with resection of the nonviable small bowel and creation of an iliacomesenteric bypass by the use of autologous saphenous vein graft. After two days, a decrease of renal function was observed (eGFR: $22 \mathrm{~mL} / \mathrm{min} / 1.73 \mathrm{~m}^{2}$ [CKD-EPI]) together with laboratory signs of ischemic hepatitis (AST 4366U/l, ALT 3942 U/L, bilirubin total $1.34 \mathrm{mg} / \mathrm{dl}$ ) and an elevated serum lactate of $9.0 \mathrm{mmol} /$ L. Nevertheless, duplex ultrasound revealed normal flow of all visceral arteries as confirmed by repeat CTA. As the primary entry tear was localized at the level of the supra-aortic vessels, primary coverage of the entry tear by thoracic endografting was impossible. The clinical situation of the patient further deteriorated, and an attempt to fenestrate the dissection flap was made. Under general anesthesia, percutaneous access of both femoral arteries
209

210 

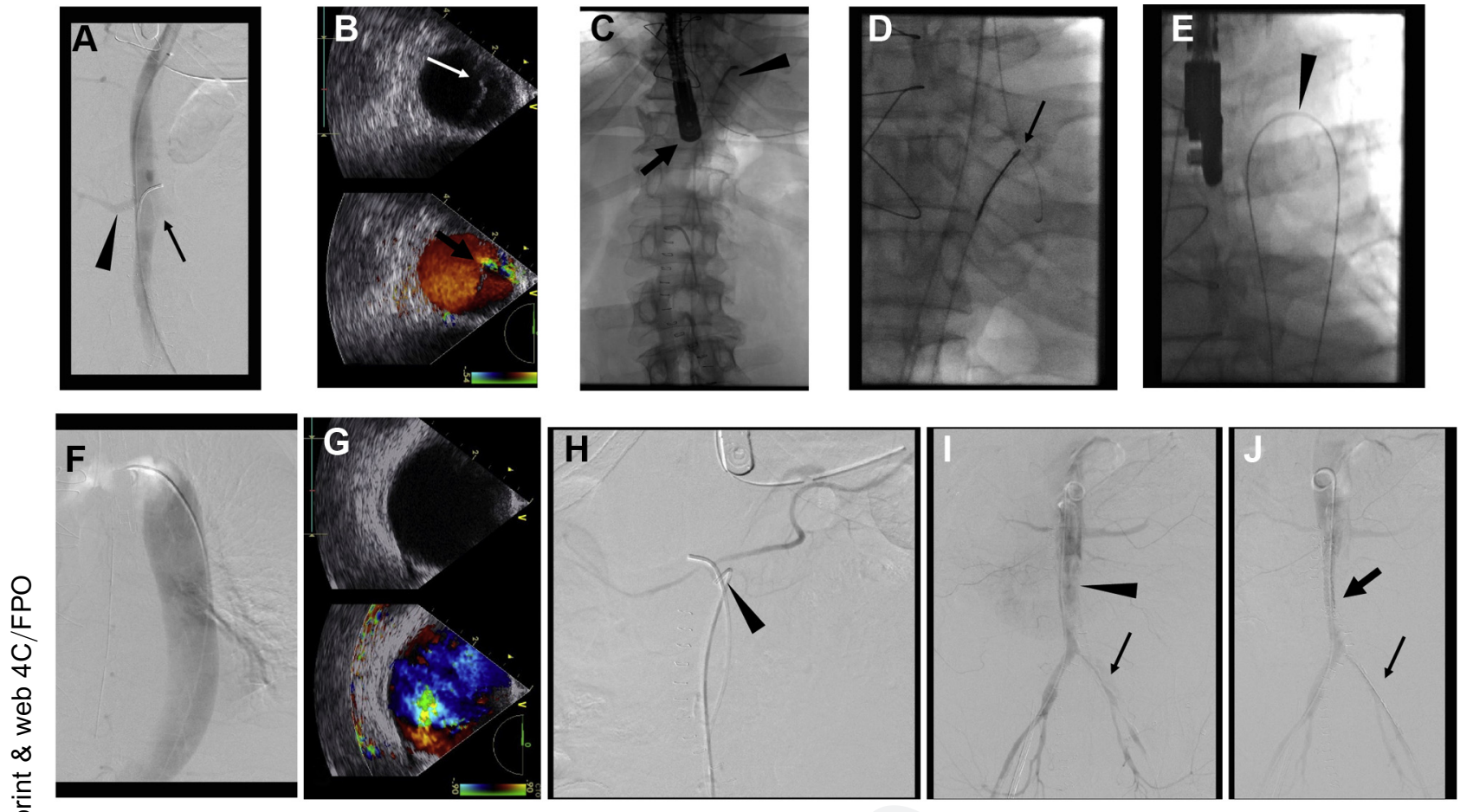

Fig. 2. (A) Angiography from the true lumen showing the right renal artery (arrowhead) without a visible connection between true and false lumen. The ostium of the left renal artery is also visible (small arrow). (B) Dynamic dissection flap visible on transesophageal echocardiography. The upper image gives good visualization of the dissection flap with a (re)-entry tear (small white arrow). The lower image uses color duplex to depict the high flow velocity through the lumen (large arrow). (C) Echocardiography-guided catheterization of the false lumen using a Cobra catheter (arrowhead), the echocardiography probe (large arrow), and echocardiography imaging. (D) Snaring of the guidewire from the false lumen (small arrow) after earlier needle perforation of the dissection flap at the level of the infrarenal aorta. (E) Throughand-through wire from the true to false lumen (arrowhead). The guidewire is gently pulled downward

was created with placement of $8 \mathrm{~F}$ sheaths. Spontaneous

Q4 catheterization of the false lumen with a 0.035 guidewire (Terumo Corporation, Tokyo, Japan) was not possible. In a first step, the dissection flap was perforated using an OUTBACK Elite Re-Entry Catheter (Cordis; Cardinal health, Baar, Switzerland) at the level of the infrarenal aorta. The fenestration hole was dilated using a $10 \times 20$ dilatation balloon. Several attempts to fenestrate the dissection flap at the level of the descending aorta using the reentry catheter or TIPS needle (Rösch-Uchida transjugular liver access set; Cook Medical, Bloomington, IN) was not possible probably due to the mobility of the dynamic dissection flap. Concomitant TEE (Vivid S6 BT11 CV System with probe 6Tc [3.0-8.0 MHz]; GE Healthcare, to cleave the dissection flap. (F) Control angiography after performing the cheese-wire technique showing complete visualization of the aortic diameter. (G) Complete disappearance of the dissection flap on echocardiography without the residual visual dissection flap on the upper image. Color duplex showing diffuse blood flow over the entire aortic diameter on the lower image. (H) Easy selective catheterization of the celiac trunk (arrowhead) after cleavage of the dissection flap. (I) Control angiography showing accumulation of the dissection flap at the level of the infrarenal aorta (arrowhead), just below the level of earlier needle perforation. Weak flow in the left iliac axis due to the obstructive character (small arrow). (J) Final control angiography after stenting of the infrarenal aorta using a Zilver vena $16 \times 60$ (large arrow) with recuperation of flow over the left iliac axis (small arrow) with normalization of pressure at the femoral level.

Chicago, IL) could detect a dynamic small re-entry tear in the dissection flap in the aortic arch, which was not visible on angiography or CTA. Under TEE guidance, the fenestration was catheterized using the position of the ultrasonography probe as a landmark. The 0.035 guidewire was advanced and snared using a goose neck snare (Amplatz Goose Neck snare kit, ev3; Plymouth, MN) and pulled into the $8 \mathrm{~F}$ sheath. After creating a through-andthrough guidewire control, a gently bilateral pull down maneuver was performed, as earlier described by Kos et al., ${ }^{6}$ up to the level of the infrarenal aorta. Control angiography documented complete expansion of the aortic lumen. No residual dissection flap could be noted on TEE. Nevertheless, at the level of the infrarenal aorta, 
accumulation of the cleaved aortic intima was noted with obstruction of the left iliac axis and disappearance of left femoral pulse. Therefore, the infrarenal aorta was stented using a Zilver vena $16 \times 60$ balloon expandable stent (Cook Medical, Bloomington, IN) with normalization of Q5 the patient's ischemic symptoms of the left leg. Finally, selective catheterization of the celiac trunk, SMA, and both renal arteries showed small caliber in spite of being patent vessels (Fig. 2). Unfortunately, due to the long-standing preinterventional visceral hypoperfusion, severe ischemia-reperfusion injury occurred, which caused further deterioration of the clinical situation. The patient died on the first postoperative day.

\section{DISCUSSION}

Open surgical treatment of type A aortic dissections remains to have a high perioperative mortality rate of $25 \% .^{8}$ Concomitant visceral malperfusion may worsen the operative outcome. ${ }^{3}$ Endovascular treatment of complicated type A aortic dissection has been described successfully in the past. ${ }^{9}$ Open repair after restoration of the malperfusion is gaining increasing interest, and some authors suggest that treating the visceral malperfusion before open central aortic repair may be beneficial. Patient-specific algorithms are being developed, in which fenestration is recommended as a first step. After awaiting resolution of the malperfusion syndrome, central aortic repair can be considered. ${ }^{1,10}$

Different techniques have been described to perforate the dissection membrane in case of complicated aortic type B dissection. The use of TIPS needles for percutaneous fenestration of the intimal flap in the dissected aorta with malperfusion has been well described. Successful results are also reported with the use of commercially available reentry catheters. ${ }^{4,5}$

It is recommended that these maneuvers are being performed with the use of additional imaging techniques, including intravascular ultrasound (IVUS) for percutaneous fenestration of the intimal flap in the dissected aorta with malperfusion using a TIPS needle. ${ }^{4,5}$ If the false lumen is large enough, perforation can be performed based on preoperative CTA and with fluoroscopic guidance. ${ }^{711}$ TEE has already been widely used in guiding complex percutaneous cardiovascular interventions such as the repair of structural heart defects or valvular diseases. ${ }^{12}$ TEE has also an important role in the diagnosis of aortic dissection and aneurysms. Angiography, TEE, and IVUS are able to detect main entry tears of aortic dissection. TEE and IVUS $(P<0.005$ each $)$ have a higher sensitivity in detecting secondary entries than angiography. ${ }^{13}$ We encountered problems with both TIPS needle and reentry catheter probably due to the mobility of the dissection membrane. As many entry tears are not visualized on CTA or angiography, TEE can be used to screen the descending aorta on possible small dynamic entry tears and to guide during the catheterization of this entry tear.

Finally, after performing the cheese-wire maneuver, an accumulation of the cleaved aortic intima caused outflow obstruction in our case. In the cases originally described by Kos et al., intima accumulation was also described in one out of four patients. ${ }^{6}$ This patient was treated in the acute phase. This suggest that the "cheese-wire" technique, as earlier described, does not always slit the dissection membrane, but in certain cases (both early and midterm [4 weeks]), pull down of the aortic intima can be caused, requiring additional stenting to resolve outflow obstruction. This phenomenon suggests that if this cheese-wire technique is performed, it should be done up to below the level of the renal arteries to allow additional stenting when needed.

\section{CONCLUSION}

Complicated type A dissection often has a fatal Q6 outcome, and no time should be lost to restore organ reperfusion. The described technique using TEE to screen for secondary entries is a useful tool when dissection flap perforation with the use of needles is impossible. One possible treatment option is the usage of the "cheese-wire" technique. In the acute phase, this maneuver should always be done beneath the level of the visceral arteries.

\section{REFERENCES}

1. Kamman AV, Yang B, Kim KM, et al. Visceral malperfusion in aortic dissection: the Michigan experience. Semin Thorac Cardiovasc Surg 2017;29:173-8.

2. Riambau V, Böckler D, Brunkwall J, et al. Management of descending thoracic aorta diseases: clinical practice guidelines of the European Society for Vascular Surgery (ESVS). Eur J Vasc Endovasc Surg 2017;53:4-52.

3. Erbel R, Aboyans V, Boileau C, et al., ESC Committee for Practice Guidelines. 2014 ESC guidelines on the diagnosis and treatment of aortic diseases: Document covering acute and chronic aortic diseases of the thoracic and abdominal aorta of the adult. The Task Force for the diagnosis and treatment of aortic diseases of the European Society of Cardiology (ESC). Eur Heart J 2014;35:2873-926.

4. Chavan A, Hausmann D, Dresler C, et al. Intravascular ultrasound-guided percutaneous fenestration of the intimal flap in the dissected aorta. Circulation 1997;96:2124-7.

5. Saket RR, Razavi MK, Padidar A, et al. Novel intravascular ultrasound-guided method to create transintimal arterial communications: initial experience in peripheral occlusive disease and aortic dissection. J Endovasc Ther 2004;11: 274-80. 
6. Kos S, Gürke L, Jacob AL. A novel fenestration technique for abdominal aortic dissection membranes using a combination of a needle re-entry catheter and the "cheese-wire" technique. Cardiovasc Intervent Radiol 2011;34:1296-302.

7. Mufty H, Katsargyris A, Houthoofd S, et al. Technical Note: dissection flap perforation with use of a TIPS-needle during fenestrated endografting for post-dissection thoracoabdominal aneurysms. Cardiovasc Intervent Radiol 2018;41:964-7.

8. Trimarchi S, Nienaber CA, Rampoldi V, et al., International Registry of Acute Aortic Dissection Investigators. Contemporary results of surgery in acute type A aortic dissection: the International Registry of Acute Aortic Dissection experience. J Thorac Cardiovasc Surg 2005;129:112-22.

9. Zimpfer D, Czerny M, Kettenbach J, et al. Treatment of acute type a dissection by percutaneous endovascular stent-graft placement. Ann Thorac Surg 2006;82:747-9.
10. Patel HJ, Williams DM, Dasika NL, et al. Operative delay for peripheral malperfusion syndrome in acute type A aortic dissection: a long-term analysis. J Thorac Cardiovasc Surg 2008; 135:1288-95.

11. Wolfschmidt F, Hassold N, Goltz JP, et al. Aortic dissection: accurate subintimal flap fenestration by using a reentry catheter with fluoroscopic guidance-initial single-institution experience. Radiology 2015;276:862-72.

12. Silvestry FE, Kerber RE, Brook MM, et al. Echocardiography-guided interventions. J Am Soc Echocardiogr 2009;22: 213-31.

13. Koschyk DH, Nienaber CA, Knap M, et al. How to guide stent-graft implantation in type B aortic dissection? Comparison of angiography, transesophageal echocardiography, and intravascular ultrasound. Circulation 2005;112(9 Suppl):I260-4. 